\title{
Infant fever trends following the launch of the meningococcal B vaccine in the UK
}

\author{
Sally Harcourt ${ }^{\star 1}$, Roger Morbey ${ }^{1}$, Chris Bates ${ }^{2}$, Helen Carter ${ }^{1}$, Shamez Ladhani ${ }^{3}$, \\ Alex J. Elliot ${ }^{1}$ and Gillian E. Smith ${ }^{1}$
}

${ }^{1}$ Real-time Syndromic Surveillance Team, Public Health England, Birmingham, United Kingdom; ${ }^{2}$ The Phoenix Partnership (TPP), Leeds, United Kingdom; ${ }^{3}$ Public Health England Immunisation, Hepatitis and Blood Safety Department, London, United Kingdom

\section{Objective}

To use syndromic surveillance data to assess whether there has been an increase in GP fever consultations since the inclusion of the meningococcal B (MenB) vaccine in the UK vaccination schedule.

\section{Introduction}

From 1 September 2015, babies in the United Kingdom (UK) born on/after 1 July 2015 became eligible to receive the MenB vaccine, given at 2 and 4 months of age, with a booster at 12 months. ${ }^{1}$ Early trials found a high prevalence of fever (over $38^{\circ} \mathrm{C}$ ) in babies given the vaccine with other routine vaccines at 2 and 4 months. We used syndromic surveillance ${ }^{2}$ data to assess whether there had been increased family doctor (general practitioner (GP)) consultations for fever in young infants following the introduction of the vaccine.

\section{Methods}

GP consultations for fever in infants aged under 1 year were extracted from The Phoenix Partnership (TPP) ResearchOne database (400 GP surgeries in England). ${ }^{3}$ Data were stratified by week of age over the period 1 September 2015 to 30 November 2015 and 1 December 2015 to 29 February 2016. Fever consultation rates (per 100,000 registered practice population in the database) were compared to the same 3 month periods of the previous 5 years (2010-14) using incident rate ratios (IRR). Pre- and post-vaccination consultation rates were applied to the England 0-26 week population to estimate excess fever consultations.

\section{Results}

Between 1 September and 30 November 2015 the average daily fever consultation rate for infants aged 0-26 weeks was 4.72/100,000; the incident rate ratio was $1.46(95 \% \mathrm{CI}, 1.09-1.92)$. In the $7-10$ week age group the average daily fever consultation rate was 7.79/100,000. The incidence rate was 2.68 times higher than in previous years (95\% CI, 1.42-4.94).

Between 1 December 2015 and 29 February 2016 the average daily consultation rate for infants aged 0-26 weeks was 6.19/100,000. The incidence rate was 1.49 times higher than in the same 3 month period of previous years (95\% CI, 1.16-1.90). In infants aged 7-10 weeks the average daily consultation rate was $8.44 / 100,000$ and the incidence rate was 1.83 times higher than previous years (95\% CI 1.03-3.16).

Between 1 September 2015 and 29 February 2016 there were an estimated additional 959 fever consultations for infants aged 0-26 weeks to English family doctors.

\section{Conclusions}

We have demonstrated an innovative use of syndromic surveillance to quickly and easily assess the impact on healthcare seeking behaviour for infants with fever following the introduction of a new vaccination into the routine vaccination programme in England. Our study provides reassurance that in infants aged 0-26 weeks there was no marked increase in consultations following the introduction of the new MenB vaccination. However, in some age groups below
0-26 weeks there was an increase in healthcare seeking behaviour for fever, in particular, the 7-10 week age group which includes infants aged 8 weeks receiving their first vaccination. Other age groups also demonstrated increased fever consultations during these two periods, albeit at less significant levels. We will analyse data for the full year from 1 September 2015 to further explore these findings, investigate potential confounders and assess trends since vaccine introduction.

\section{Keywords}

Syndromic surveillance; Meningococcal B; Vaccination

\section{Acknowledgments}

The authors would like to thank the TPP ResearchOne Project Committee for permission to use the ResearchOne database for this study. We also thank Mr Paul Loveridge of the Public Health England (PHE) Realtime Syndromic Surveillance Team (ReSST) for technical expertise.

\section{References}

1. Public Health England [Internet]. MenB vaccination: introduction from September 2015 [updated 22 June 2015; cited 24 August 2016]. Available from: https://www.gov.uk/government/publications/menbvaccination-introduction-from-1-september-2015

2. Public Health England [Internet]. Syndromic surveillance: systems and analyses [updated 15 January 2015; cited 24 August 2016]. Available from: https://www.gov.uk/government/collections/syndromicsurveillance-systems-and-analyses

3. ResearchOne [Internet]. Transforming data into knowledge. [cited 24 August 2016]. Available from: http://www.researchone.org/

\section{*Sally Harcourt}

E-mail: sally.harcourt@phe.gov.uk 